\title{
A preliminary case study of the effect of shoe-wearing on the biomechanics of a horse's foot
}

Olga Panagiotopoulou, Jeffery W Rankin, Stephen M Gatesy, John R Hutchinson

Horse racing is a multi-billion-dollar industry that has raised welfare concerns due to injured and euthanized animals. Whilst the cause of musculoskeletal injuries that lead to horse morbidity and mortality is multifactorial, pre-existing pathologies, increased speeds and substrate of the racecourse are likely contributors to foot disease. Horse hooves have the ability to naturally deform during locomotion and dissipate locomotor stresses, yet farriery approaches are utilised to increase performance and protect hooves from wear. Previous studies have assessed the effect of different shoe designs on locomotor performance; however, no biomechanical study has hitherto measured the effect of horseshoes on the stresses of the foot skeleton in vivo. This preliminary study introduces a novel methodology combining three-dimensional data from biplanar radiography with inverse dynamics methods and finite element analysis (FEA) to evaluate the effect of a stainless steel shoe on the function of a Thoroughbred horse's forefoot during walking. Our preliminary results suggest that the stainless steel shoe shifts craniocaudal, mediolateral and vertical GRFs at mid-stance. We document a similar pattern of flexion-extension in the PIP (pastern) and DIP (coffin) joints between the unshod and shod conditions, with slight variation in rotation angles throughout the stance phase. For both conditions, the PIP and DIP joints begin in a flexed posture and extend over the entire stance phase. At midstance, small differences in joint angle are observed in the PIP joint, with the shod condition being more extended than the unshod horse, whereas the DIP joint is extended more in the unshod than the shod condition. We also document that the DIP joint extends more than the PIP after mid-stance and until the end of the stance in both conditions. Our FEA analysis, conducted solely on the bones, shows increased von Mises and Maximum principal stresses on the forefoot phalanges in the shod condition at mid-stance, consistent with the tentative conclusion that a steel shoe might increase mechanical loading. However, because of our limited sample size none of these apparent differences have been tested for statistical significance. Our preliminary study illustrates how the shoe may influence the dynamics and mechanics of a Thoroughbred horse's forefoot during slow walking, but more research is needed to quantify the effect of the shoe on the equine forefoot during the whole stance phase, at faster speeds/gaits and with more individuals as 
well as with a similar focus on the hind feet. We anticipate that our preliminary analysis using advanced methodological approaches will pave the way for new directions in research on the form/function relationship of the equine foot, with the ultimate goal to minimise foot injuries and improve animal health and welfare. 
1 A preliminary case study of the effect of shoe-wearing on the biomechanics of a horse's foot 2

3 Olga Panagiotopoulou ${ }^{1,2}$, Jeffery W. Rankin ${ }^{1}$, Stephen M. Gatesy ${ }^{3}$, John R. Hutchinson ${ }^{1,2}$

4

$5 \quad{ }^{1}$ Structure \& Motion Laboratory, Department of Comparative Biomedical Sciences, Royal 6 Veterinary College, Hatfield, United Kingdom.

7 2Moving Morphology \& Functional Mechanics Laboratory, School of Biomedical Sciences, 8 Faculty of Medicine and Biomedical Sciences, The University of Queensland, Brisbane, Australia $9{ }^{3}$ Department of Ecology and Evolutionary Biology, Brown University, Providence, Rhode 10 Island, USA.

12 Corresponding Authors:

Dr. Olga Panagiotopoulou Moving Morphology \& Functional Mechanics Laboratory, School of Biomedical Sciences, The University of Queensland, Room 433-Otto Hirschfeld Building (81), Brisbane, QLD 4072, Australia.

17 o.panagiotopoulou@uq.edu.au

Prof. John R Hutchinson

Structure \& Motion Laboratory, The Royal Veterinary College, Hawkshead Lane, North 21 Mymms, Hatfield, AL9 7TA, United Kingdom.

22 jhutchinson@rvc.ac.uk 
Abstract

Horse racing is a multi-billion-dollar industry that has raised welfare concerns due to injured and euthanized animals. Whilst the cause of musculoskeletal injuries that lead to horse morbidity and mortality is multifactorial, pre-existing pathologies, increased speeds and substrate of the racecourse are likely contributors to foot disease. Horse hooves have the ability to naturally deform during locomotion and dissipate locomotor stresses, yet farriery approaches are utilised to increase performance and protect hooves from wear. Previous studies have assessed the effect of different shoe designs on locomotor performance; however, no biomechanical study has hitherto measured the effect of horseshoes on the stresses of the foot skeleton in vivo. This preliminary study introduces a novel methodology combining three-dimensional data from biplanar radiography with inverse dynamics methods and finite element analysis (FEA) to evaluate the effect of a stainless steel shoe on the function of a Thoroughbred horse's forefoot during walking. Our preliminary results suggest that the stainless steel shoe shifts craniocaudal, mediolateral and vertical GRFs at mid-stance. We document a similar pattern of flexionextension in the PIP (pastern) and DIP (coffin) joints between the unshod and shod conditions, with slight variation in rotation angles throughout the stance phase. For both conditions, the PIP and DIP joints begin in a flexed posture and extend over the entire stance phase. At mid-stance, small differences in joint angle are observed in the PIP joint, with the shod condition being more extended than the unshod horse, whereas the DIP joint is extended more in the unshod than the shod condition. We also document that the DIP joint extends more than the PIP after mid-stance and until the end of the stance in both conditions. Our FEA analysis, conducted solely on the bones, shows increased von Mises and Maximum principal stresses on the forefoot phalanges in the shod condition at mid-stance, consistent with the tentative conclusion that a steel shoe might increase mechanical loading. However, because of our limited sample size none of these apparent differences have been tested for statistical significance. Our preliminary study illustrates how the shoe may influence the dynamics and mechanics of a Thoroughbred horse's forefoot during slow walking, but more research is needed to quantify the effect of the shoe on the equine forefoot during the whole stance phase, at faster speeds/gaits and with more individuals as well as with a similar focus on the hind feet. We anticipate that our preliminary 
63

64

65

66

67

68

analysis using advanced methodological approaches will pave the way for new directions in research on the form/function relationship of the equine foot, with the ultimate goal to minimise foot injuries and improve animal health and welfare.

\section{Introduction}

Horse racing is a multi-billion-dollar, worldwide industry in which the welfare of the horses is of paramount importance. Musculoskeletal injuries are both a common cause of economic loss within the industry and a major welfare concern due to the resulting morbidity and mortality (McKee 1995; Jeffcott et al., 1982; Clegg 2011; Bailey et al., 1999). The cause of musculoskeletal injuries is multifactorial: pre-existing pathologies, increased speeds, and track surfaces are all recognised as contributing factors (Parkin et al., 2004; Cogger et al., 2006; Foote et al., 2011; Clegg 2011). Horses have evolved to only maintain their third digit, which ends in a rigid hoof capsule and is functionally adapted to fast speeds (Dyce et al., 2010). The hoof and the interphalangeal joints receive most of the impact loads when the foot hits the ground (Dyhre-Poulsen et al., 1994) and at fast speeds these loads can exceed 2.5 times the horse's body weight (Witte et al., 2004). Under load-bearing conditions, the distal and coronary borders of the hooves expand (Colles 1989); the dorsal hoof wall rotates caudoventrally about the third digit and the heel expands between 2-4mm (Jordan et al., 2001).

Farriery (horseshoe design) approaches in both domestic and racehorses have been used since the domestication of horses to protect hooves from wear and to allow manipulation of the shape of the foot to improve performance and enhance biomechanical function. Nevertheless, different horseshoe materials have varying effects on horses' feet due to their wide range of weight, toe angle, frictional and damping properties and their interaction with foot trimming (Roepstorff, Johnston \& Drevemo 1999; Pardoe et al., 2001; van Heel et al., 2005; van Heel, van Weeren \&, Back 2006; Heidt et al., 1996, Willemen, Savelberg \& Barneveld 1998). Previous in vivo studies in horses have shown that an elevation of the hoof due to the presence of the shoe increases the pressure within the distal interphalangeal joint, which may account for an increase of bone stresses that can enhance the development of degenerative joint diseases (Roepstorff, Johnston \& Drevemo 1999). Although no biomechanical study to date has quantified bone stresses of the horse forefoot in the shod and unshod conditions in vivo, Moyer and Anderson (1975) hypothesised that increased loading due to farriery can increase stresses 
94 on the horse foot and lead to injuries (Moyer \& Anderson 1975). In addition, an ex vivo analysis by Ault et al., (2015) recorded significant increases in the strain of the superficial digital flexor tendon (SDFT) and the suspensory ligament of shod horses, further supporting the inference that shoes disrupt the natural ability of horses' feet to maintain tendon (and perhaps other tissue) strains at lower levels.

Despite the likelihood that shoes impact equine digit function, current knowledge of the relationships between foot function, farriery approaches and musculoskeletal injury is limited, partly due to the lack of an established in vivo experimental protocol for studying foot dynamics and mechanics. Finite element analysis (FEA) is a numerical technique well entrenched in equine biomechanics as a tool to measure deformation (stress, strain) in complex continuous systems (such as the hoof), by dividing them into sub-regions of finite size (elements) using linear ordinary differential equations (Hutton 2003). With FEA, scientists have managed to study the deformations of anatomically deep structures of the equine distal foot in shod and unshod conditions (Harrison et al., 2014; Hinterhofer, Stanek \& Haider 2001; Hinterhofer, Stanek \& Binder 1998; Bowker et al., 2001; Salo, Runciman \& Thomason 2009; O'Hare et al., 2013; Thomason et al., 2001, 2002, 2005; McClinchey, Thomason \& Jofriet 2003; Collins et al., 2009; Douglas et al., 1998). These studies have enhanced our understanding on how the equine digit deforms under load-bearing, but more robust in vivo data and subject-specific models are needed to fully characterize how the equine distal limb's functional environment relates to disease. This requires combining accurate joint motion and ground reaction force (GRF) data from a synchronised time sequence with subject specific bone geometry. Here we show how a combination of different techniques can be used to obtain these data to generate high fidelity in vivo FEA results.

A common approach for researchers to measure joint motion in horses is the attachment of motion analysis markers on the skin overlying bony structures. This approach introduces errors, due to artefacts from skin and hoof motion, which can be as large as the actual joint motion (Reinschmidt et al., 1997; Roach et al., 2015). One alternative to surface skin markers is the surgical implantation of intra-cortical bone pins into the limb bones, but this methodology is highly invasive (e.g. Clayton et al., 2004, 2007a; van Weeren, van den Bogert \& Barneveld 1990; Chateau, Degueurce \& Denoix 2004). Although these pins can more accurately quantify bone motion, their invasiveness may affect the natural function/behaviour of the joints 
125 (Lundberg et al., 1989) and are inappropriate to use when studies require a large number of

126 horse participants. Fortunately, a new alternative technology using biplanar radiography, 127 commonly referred to as X-ray Reconstruction of Moving Morphology or XROMM, has been 128 developed that can be used to accurately characterize the three-dimensional (3D) motion of 129 joints (Brainerd et al., 2010; Gatesy et al., 2010).

130 XROMM combines bi-planar fluoroscopic images to track dynamic functions such as 131 trotting, which enables precise measurements of joint motion without artefacts from soft tissue 132 motion (e.g., Miranda et al., 2013). By acquisition of fluoroscopic images in two planes and 133 with the assistance of specialised software, the images can be combined to track motion of 134 individual skeletal elements in three dimensions. Thus, motion can be assessed in vivo, without 135 the requirement for attachment of any device to the skin or into the bones. Natural behaviour 136 can be measured in a manner not possible with other techniques and with minimal risk to the 137 animal/participant, while keeping radiation doses reasonably low. To date, the XROMM 138 marker-based (Astley \& Roberts 2011; Baier \& Gatesy 2013; Brainerd, Moritz \& Ritter 2015; 139 Brainerd et al., 2010; Camp, Roberts \& Brainerd 2015; Camp \& Brainerd 2014; Dawson et al., 140 2011; Gidmark et al., 2013; Heers et al., 2016; Kambic, Roberts \& Gatesy 2014, 2015; 141 Nowroozi \& Brainerd 2013) and markerless (Baier, Gatesy and Dial 2013; Falkingham \& 142 Gatesy 2014; Gatesy et al., 2010; Nyakatura \& Fischer 2010) technology has been used to 143 study diverse behaviours such as the limb kinematics of frogs, birds, bats and dogs; jaw kinematics during feeding in fish, pigs, birds and bats and rib kinematics of breathing in lizards.

This study presents a novel method that combines three-dimensional data from XROMM (Brainerd et al., 2010; Gatesy et al., 2010), inverse dynamics methods, and finite element analysis to perform a preliminary investigation of the effect of a stainless steel shoe on the function of one Thoroughbred horse's foot during walking. The intent of this work is not to draw clinical conclusions on the effect of the shoe on equine foot mechanics. Instead, we present a methodological approach that can be used in future research to study the effect of different shoe designs on foot mechanics and potentially inform the design of new shoes that can improve locomotor performance while maintaining the integrity of musculoskeletal structures.

\section{Materials \& Methods}




\section{Subjects}

157 One Thoroughbred healthy male adult horse (540 kg body mass) from the Royal Veterinary 158 College (RVC) participated in the study. The horse had previously been trained for and 159 participated in locomotor studies in the laboratory. Fifteen minutes of training were provided for 160 the horse to adapt to the experimental setup. The study was reviewed and approved by the Royal 161 Veterinary College's Ethics and Welfare Committee (approval number URN 2011 1094).

\section{Data Collection}

163 Each trial lasted two to four seconds, during which the horse was led across a custom164 designed platform (Figure 1A). A custom-designed platform rather than a treadmill was used for

165

166

167

168

169

170

171

172

173

174

175

176

177

178

179

180

181

182

183

184

185

186

this study because our methodological approach for the in vivo estimation of the intersegmental forces for FEA required accurate ground reaction force (GRF) measurements. Accurate measurements of all GRF components could not be obtained using any available treadmill.

A Sony HDR (Sony, London, UK) high definition video camera was placed perpendicular to the platform to approximate walking speed $(25 \mathrm{~Hz})$. To obtain foot kinematics, two custom x-ray fluoroscopes (RSTechnics, Netherlands; refurbished Phillips systems, $36 \mathrm{~cm}$ intensifier; $\leq 110 \mathrm{kV}$, $\leq 3 \mathrm{~mA}$ ) were retrofitted with two AOS high-speed digital cameras (AOS Technologies AG, Switzerland) to acquire biplanar fluoroscopy images at $250 \mathrm{~Hz}$ of the horse's feet as it walked through an undistorted and calibrated capture volume ( $\sim 30 \mathrm{~cm}$ per cube edge) located on the force plate.

Standard videos introduce geometric distortion to the images due to lens misalignments (Dobbert 2005) and the fluoroscopes create nonlinear pincushion and spatial artefacts (Wang and Blackburn 2000), which degrade image quality. To remove this distortion, we imaged a standardized sheet of perforated metal grid $(1.6 \mathrm{~mm}$ thick with $3 \mathrm{~mm}$ diameter holes, spaced $4 \mathrm{~mm}$ apart in a $60^{\circ}$ staggered pattern) (RS Components Ltd, UK) in front of the intensifiers and captured the grid images at 32 frames for 1 second. The exposure setting for the two intensifiers during the collection of the grid images was respectively set at $57 \mathrm{kv}, 38 \mathrm{~mA}$ and $50 \mathrm{kv}, 19 \mathrm{~mA}$.

To correct the distorted video images and the calibration cube images, we created undistortion files from the standardised reference grid images using the XrayProject MatLab (Mathworks Inc. Natick, Massachusetts, USA) protocol (www.xromm.org). By using the reference grid images of each intensifier as templates, we created a uniform set of squares and applied it to all video images (Brainerd et al., 2010). The distortion correction algorithm compared the spacing 
187 between holes in the fluoroscope image with the idealized spacing and calculated a 188 transformation matrix for correcting all video images. We used a local weighted mean (LWM) 189 distortion correction algorithm, implemented in MatLab.

190 To calibrate the video images and generate a DLT algorithm in order to create "virtual 191 cameras" in the Maya virtual 3D space for rotoscopy of the horse's foot, we followed the 192 calibration process described by Brainerd et al., 2010. We placed a custom-designed calibration 193 cube with 64 steel $3 \mathrm{~mm}$ diameter spheres as calibration points within the field of view of the two 194 intensifiers and recorded the calibration images at $32 \mathrm{~Hz}$ for 1 second (Supplementary Figure S1) 195 (Brainerd et al., 2010). The calibration cube consisted of acrylic sheets of uniform thickness $196(5.42 \mathrm{~mm})$, on which we drilled 16 holes of $3 \mathrm{~mm}$ diameter in a square pattern of $65 \mathrm{~mm}$ 197 separation. Nylon pillars served as spacers between the tiers. The exposure settings for the two 198 intensifiers during the collection of the calibration cube images were set at $46 \mathrm{kv}, 39 \mathrm{~mA}$ and $71 \mathrm{kv}, 11 \mathrm{~mA}$.

200 The undistorted images of the calibration cubes for each camera were used as templates to 201 calibrate all video images using protocols established by Brainerd et al., 2010. The undistorted 202 calibration cubes were digitised by manually selecting the centroid of a minimum of 12 calibration cube beads that could be seen in both cameras using MatLab. Digitizing was repeated until a calibration coefficient of low residuals $(<0.3)$ was given for each camera. The resulting direct linear transformations (DLT) were used to optimise the focal point and axes of the cameras. The camera data were then imported to Maya software (Autodesk, San Rafael, California, USA) to recreate a virtual scene with similar coordinates to the experiment (Brainerd et al., 2010).

Exposure settings were set to $69 \mathrm{kV}, 53 \mathrm{~mA}$ and $72 \mathrm{kV}, 54 \mathrm{~mA}$ for the two sources, which were placed on the left side of the platform. Each intensifier was placed 2 metres from its corresponding x-ray source on the right side of the platform such that the beams were oriented horizontally at approximately $90^{\circ}$ to one another (Figure 1A).

Kinetic data were collected simultaneously at a rate of $1000 \mathrm{~Hz}$ using a forceplate $(60 \times 90 \mathrm{~cm}$ with Hall Effect sensors, 2000lb maximum vertical force; AMTI, Watertown, MA, USA). Prior to analysis, the forceplate data were low-pass filtered using a $4^{\text {th }}$ order zero-lag Butterworth filter with a cutoff frequency of $15 \mathrm{~Hz}$. All data were synchronized to the fluoroscope system. 
217 The unshod horse was guided 344 times across the experimental platform over a period of two

218 weeks. Following the end of the experiments for the unshod condition, the horse's forefeet 219 received mild trimming and were each fitted with a stainless steel shoe with toe clips (5 inches 220 wide) and 6 nails. The identical procedure was then followed to guide the shod horse over the 221 platform 65 times on a subsequent day. The difference in trial numbers between the unshod (344 222 strides) and the shod (65 strides) conditions was due to the large number of spatially incomplete 223 data for the former. Strides that were spatially incomplete (i.e., the right forefoot only stepped 224 partially within the capture volume) and/or unsteady (i.e., with evident deceleration and 225 acceleration following observation of the video images during data collection) were excluded 226 from further analysis. Four steps from the shod and four steps from the unshod right forefoot that 227 were spatially complete and steady were processed using the markerless XROMM (X-ray 228 Reconstruction of Moving Morphology: Brainerd et al., 2010; Gatesy et al., 2010) workflow to 229 construct a model and obtain 3D joint rotations and translations. The limited number of steps per 230 conditions is a limitation of the XROMM approach when used in live animal studies for species 231 as large as a Thoroughbred horse. For a step to be valid during the XROMM procedure, the 232 animal has to step within the calibrated field of view without deviation. If there were minimal 233 deviations from the capture volume, we were unable to visualise the distal right forefoot in both 234 cameras in order to extract the 3D joint kinematics.

235

\section{Model Construction}

The horse was euthanized at the end of the experiment for unrelated studies and its right forelimb was removed and frozen $\left(-20^{\circ} \mathrm{C}\right)$. Computed tomography scans (GE Lightspeed 16detector unit; General Electric) were used to obtain the three dimensional (3D) skeletal geometry of the horse's forefoot (slice thickness $0.625 \mathrm{~mm}, 0.460$ pixels $\mathrm{mm}^{-1}, 512 \times 512$ pixel images, 620 slices). These data were then processed to extract solid 3D polygonal mesh objects in Mimics (version 16.0; Materialise, Inc, Leuven, Belgium) and then imported into Maya (Autodesk, San Rafael, California, USA) to construct the biomechanical models' segments (Figure 1B). Four segments were defined: the metacarpus (MC), first phalanx (P1), intermediate phalanx (P2) with the navicular bone and the distal phalanx (P3). An articulated skeleton was then created by hierarchically linking these segments (Gatesy et al., 2010) into a kinematic chain representing 
247 the metacarpophalangeal (MCP), proximal interphalangeal (PIP) and distal interphalangeal (DIP) 248 joints (Figure 2).

249 Joint orientations and positions were defined by first positioning all bone segments into a 250 neutral anatomical pose (forefoot lying fully horizontally). Cylinders were then visually fit to the 251 joint surfaces (i.e., the distal epiphyses of the MC, P1 and P2) to identify the axes of joint 252 rotations. These locations were confirmed when manual manipulation of the virtual joint resulted 253 in a natural motion where adjacent bones did not interpenetrate each other. Dissected cadaveric 254 specimens and plastic models were used to further assess joint locations and positions. 255 Transformations between coordinate systems were defined using a Z (green axis),

$256 \mathrm{Y}^{\prime}$ (yellow axis) and $\mathrm{X}^{\prime \prime}$ (red axis) Cardan rotation, respectively representing abduction257 adduction, flexion-extension and long axis rotation (Figure 2). Axes were defined so that positive 258 joint angles represented adduction, flexion and external long axis rotation.

259

260

\section{Markerless XROMM}

261

Trajectories for each joint were quantified using established protocols (Brown University, 262 USA; www.xromm.org) for scientific rotoscoping (markerless XROMM) (Gatesy et al., 2010; Baier \& Gatesy 2013; Baier et al., 2013; Nyakatura \& Fischer 2010). In brief, markerless XROMM is a technique that allows one to quantify 3D motion by animating model segments (i.e., 3D polygonal mesh objects) to match postures observed in experimental x-ray video images (Figure 1B). For each experimental x-ray trial, the horse foot model was aligned with the bone $\mathrm{x}$ ray silhouettes in undistorted and calibrated video images using the anatomical features of each bone as reference guides (Supplementary movies 1 \& 2). Joint transformations (i.e., joint rotations and translations) were then extracted from the model for the MCP, PIP and DIP joints (Figure 2). The MCP kinematic data were excluded from further analysis because the midshaft and proximal epiphysis were out of the field of view for most of the stride.

All steps for shod $(n=4)$ and unshod $(n=4)$ conditions were used to measure joint kinematics and foot kinetics, but a single representative step was selected for each condition ( $\operatorname{shod~} n=1$; unshod $n=1$ ) for the subsequent mid-stance inverse dynamics calculations and FEA used to estimate bone stresses in the right forefoot digit.

\section{Inverse Dynamics}


278

279

280

281

282

283

284

285

286

287

288

289

290

291

292

293

294

295

296

297

298

299

300

301

302

303

304

305

306

307

308

Inverse dynamics methods were used to calculate the intersegmental forces (required for estimating bone stresses) at mid-stance for the two steps selected for FEA (shod and unshod conditions). Larger loads can occur at other points during the stance phase but the focus of this study is methodological and for simplicity we modelled solely mid-stance.

To perform the analysis, the skeletal model created for scientific rotoscoping was recreated in Software for Interactive Musculoskeletal Modeling (SIMM; Musculographics, California, USA). To create an exact replica of the original model, bone geometry was exported directly from Maya and imported into SIMM, where they were reassembled by reproducing the original joint structure (i.e., number of joints and degrees of freedom). The model was then imported into OpenSim (Delp et al., 2007), which has a built-in routine to perform inverse dynamics.

Mid-stance was defined as the point halfway between foot strike and toe-off gait events, which were determined from the vertical GRF data. Mid-stance joint angles were exported directly from the XROMM workflow and used to position the model in OpenSim. Mid-stance GRFs, obtained from the synchronized force plate data, were transformed into the same reference frame as the OpenSim foot model and then applied to the distal phalanx (P3). Data integrity between the motion and force data were verified by visually inspecting the location of the centre of pressure (CoP) (from forceplate data) relative to the foot placement (from XROMM kinematics) using OpenSim. Because the distal segments are small (i.e., low mass) and walking is a slow motion (i.e., low joint accelerations), gravity and inertial forces were assumed to be negligible relative to the forces resulting from the applied mid-stance GRFs. Thus, mass and inertial properties for all segments were not included in the analysis. OpenSim's inverse dynamics and joint reaction force routines were then used to calculate the intersegmental joint forces and moments acting on the segments at each joint. These data were expressed in the local frame of the segment and used as inputs into the FEA.

\section{Finite Element Analysis}

For each phalanx (P1, P2 [including the navicular bone] and P3), a separate finite element model was created in Abaqus/CAE, software version 6.13 (Dassault Systemes Simulia Corp, Providence, Rhode Island, USA). The corresponding intersegmental forces from the inverse dynamics analysis were applied to each bone independently and stress was determined using the Abaqus/Standard implicit direct default solver. 
$\underline{\text { Bone Models }}$

Each 3D solid bone mesh representing a segment from the OpenSim model was imported into 3-Matic 9.0 software (Materialize Inc., Leuwen, Belgium) and converted into a volumetric mesh file of continuum linear tetrahedral elements of type C3D3. Each volumetric mesh file (preserving the coordinate systems of each segment as defined during the inverse dynamics analysis) was then imported into Abaqus/CAE 6.13 FEA software and converted into 10 node quadratic hybrid elements of type $\mathrm{C} 3 \mathrm{D} 10 \mathrm{H}$. The element nominal size for all models was $2 \mathrm{~mm}$.

317 The P1, P2 (with the navicular) and P3 segments had 60,967; 42,401 and 35,725 elements respectively.

\section{$\underline{\text { Material Properties }}$}

Due to a lack of specific material properties data for the bones of the distal foot of horses, linear elasticity, homogeneity and isotropy were assumed for each bone model. Assumptions regarding isotropy and homogeneity should create a constant error between our models and thus do not influence bone stress comparisons between the shod and the unshod horse. We assigned a Young's modulus (E) value of $16,000 \mathrm{MPa}$ and Poisson's ratio (v) of 0.3 to the $\mathrm{P} 1$ and P2. The P3 in horses consists of dense trabeculae and was thus assigned a modulus of 10,000 MPa and Poisson's ratio of 0.3 (Rho et al., 2001; Jansová et al., 2015).

Loads and constraints

To load each bone model, we applied the intersegmental forces calculated during the inverse dynamics routine at mid-stance to the bone segment surface associated with the joint of interest. To apply each load, we selected the nodes at the entire joint surface for P1, P2 and P3 and divided the $\mathrm{x}, \mathrm{y}, \mathrm{z}$ force components uniformly across the joint surface.

Each bone model was loaded and analysed separately, using the intersegmental forces to approximate the loads arising from skeletal geometry, kinematics and kinetics. The P1 bone model was loaded at the MCP joint (Supplementary Figure S2). The P2 bone model (with the attached navicular) was loaded at the PIP joint (Supplementary Figure S2) and the P3 bone was loaded at the DIP joint (Supplementary Figure S2). The proximal and intermediate phalanges were constrained by selecting the entire joint surface at the distal end of the bones and fixing 
340 rotations and displacements about all axes. The P3 was constrained on the most anterior point of 341 the palmar part.

342 To test the effect of constraints on bone stresses, we conducted a sensitivity analysis on the P1

343

344

345

346

347

348

349

350

351

352

353

354

355

356

357

358

359

360

361

362

363

364

365

366

367

368

369 and P3 of the shod condition. The P1 was fixed to be constrained on three nodes at a location approximated as the centroid of the resultant loads (to withstand bending). The rest of the nodes on the distal joint surface were fixed solely on the direction along the long axis of the bone (to withstand compression). The P3 constraints were varied by increasing the surface area of the constrained nodes and fixing the rotations and displacements about all axes.

We visually compared von Mises, maximum principal stress (S1) and minimum principal stress (S3) patterns between the shod and unshod conditions for the P1, P2 and the navicular bone, and P3.

\section{Data analysis}

Stance phase kinetic and kinematic data were normalised to $100 \%$ stance phase duration (i.e. ground contact time). Descriptive statistics were used to quantify the walking speed within the shod $(n=4)$ and the unshod $(n=4)$ conditions. A cross-correlation analysis was conducted to assess the correlation between the unshod and shod horse in overall mean GRF patterns (in the craniocaudal, mediolateral and vertical directions) and the overall pattern in mean angle of flexion-extension for the PIP and DIP joints across the stance phase (10-90\%). The first and last $10 \%$ of the stance phase were excluded from the kinematics data due to noise caused during markerless XROMM. Due to the small sample size (1 horse and four trials per condition), the differences in the kinematics, kinetics and bone stresses were not assessed for statistical significance.

\section{Results}

Speed Data

The mean walking speed of the shod and unshod conditions was at $0.72 \mathrm{~ms}^{-1}$ and $0.76 \mathrm{~ms}^{-1}$ respectively (Table 1). This corresponded to a Froude number - which is a dimensionless representation of movement speed (Alexander \& Jayes, 1983; Fr $=$ velocity ${ }^{2} *\left[9.81 \mathrm{~ms}^{-2} *\right.$ hip height $]^{-1}$ ) - of 0.05 for the shod condition and 0.06 for the unshod condition (Table 1), indicative 
370 of a slow walk. The footfall patterns also maintained the typical lateral sequence found in

371 walking (Supplementary Data 1).

\section{Kinetic Data}

373 The GRF data from the shod $(n=4)$ and unshod $(n=4)$ conditions during the stance phase of 374 locomotion are shown in Figure 3 and Supplementary Data 2. In all directions the force pattern 375 was quite similar between the shod and unshod conditions (Figure 3). The results from the cross376 correlation analysis assessing the correlation between the unshod and shod horse in overall GRF 377 patterns showed that there was a high positive correlation between the craniocaudal GRF 378 patterns (with a maximum correlation coefficient of 0.994 with a $2 \%$ lag of the shod pattern). 379 These results also showed a high positive correlation between the vertical GRF patterns (with a

380

381

382

383

384

385

386

387

388

389

390

391

392

393

394

395

396

397

398 399

400 maximum correlation coefficient of 0.996 with a $0 \%$ lag of the shod pattern). While there also seemed to be a large similarity in mediolateral GRF patterns, the strength of the positive correlation was lower than in the other directions (with a maximum correlation coefficient of 0.747 with an $11 \%$ lag of the shod pattern).

The craniocaudal GRF at the beginning of the stance phase was directed caudally, which then shifted cranially from mid-stance until the end of the stance phase of locomotion (Figure 3 ). The maximum cranial GRF for the shod horse was shown at approximately $75 \%$ of stance $(497 \mathrm{~N})$ and for the unshod horse at $78 \%$ of stance $(396 \mathrm{~N})$. Between $75-78 \%$ of stance, the shod horse showed on average a $21 \%$ higher craniocaudal GRF than the unshod horse, yet this difference was not assessed for significance.

The mediolateral GRF for the shod condition was directed medially throughout the whole stance phase and reached an average maximum force of $85 \mathrm{~N}$ after mid-stance. Contrastingly, the mediolateral GRF for the unshod horse was directed medially during the first $10 \%$ of the stance phase, shifted laterally until late mid-stance, and then was redirected medially until the end of the stance phase. The highest mediolateral GRF for the unshod horse was laterally directed and occurred before mid-stance ( 40-45\%), reaching approximately $100 \mathrm{~N}$.

There was a strong similarity in the vertical GRF pattern between the shod and unshod conditions during the stance phase. However, at mid-stance, the vertical GRF of the shod condition $(3195 \mathrm{~N})$ was approximately $10 \%$ higher than the unshod condition $(2888 \mathrm{~N})$.

\section{Kinematic Data}


401

402

403

404

405

406

407

408

409

410

411

412

413

414

415

416

417

418

419

420

421

422

423

424

425

426

427

428

429

430

The kinematic data for the shod $(n=4)$ and unshod $(n=4)$ conditions during the stance phase of locomotion are shown in Figure 4 and Supplementary Data 3. The results from the crosscorrelation analysis between the unshod and shod conditions showed a high positive correlation for the PIP mean joint angle (with a maximum correlation coefficient of 0.989 with a $0 \%$ lag of the shod pattern). A strong positive correlation was also found for the DIP mean joint angle (with a maximum correlation coefficient of 0.975 with a $0 \%$ lag of the shod pattern).

We describe some differences in kinematic patterns here for the shod vs. unshod conditions but it is very important to note that none of these have been tested for true statistical significance, because of the small sample sizes. Overall, in both the shod and unshod conditions, the PIP joint was in a flexed posture at the beginning of the stance between $10-40 \%$ of the stance phase and ended in an extended posture before mid-stance ( $45 \%$ of stance) until the end of the stance phase (90\%). Between 10-90\% of the stance phase, the PIP joint for the shod condition had an average range of motion (ROM) of 14 degrees. The average ROM for the unshod condition's PIP joint was estimated at an average of 13 degrees.

The maximum difference between the DIP joint angle in the shod and unshod conditions was at $40 \%$ of stance, when the unshod horse DIP approximates a neutral posture $(\sim=0)$, while the shod horse DIP exhibited a more flexed posture. At mid-stance and until the end of the stance phase, the DIP extended in both the shod and unshod conditions (Table 2).

\section{Finite Element Analysis (FEA)}

The intersegmental forces obtained from the inverse dynamics technique and applied to the different segment models (P1, P2 and P3) during the FEA are presented in Table 3. Our FE analysis showed that the shoe increased the concentration of von Mises and maximum principal stresses on the dorsal (Figure 5 and Figure S3) and palmar (Figure 6 and Figure S4) aspects of the distal (P1, P2, P3) bones of the horse's forefoot; however this possible increase has not been assessed for significance due to the small sample size. Comparisons of the principal stress patterns showed that in both conditions the dorsal (Figure S3 and Figure S5) and palmar aspects (Figure S4 and Figure S6) of all phalanges respectively underwent tension and compression.

The sensitivity analysis on the constraints for the P1 showed that fixing the constraints on the distal joint surface reduces von Mises stresses compared to fixing the centroid nodes and 
431 constraining the rest of the nodes to align with the long axis of the bone. However, in both cases

432 bending occurred craniocaudally (Figure S7).

433 Our sensitivity study on the P3 constraints showed that an increase in the surface area of the 434 constrained nodes reduces von Mises stresses dorsally (Figure S8).

435

436

437

438

439

440

441

442

443

444

445

446

447

448

449

450

451

452

453

454

455

456

457

458

459

460

461

\section{Discussion}

Our study implemented a new methodology that combines XROMM, inverse dynamics methods and FEA to quantify the effect of wearing a stainless steel shoe on the biomechanics of the right forefoot of a Thoroughbred horse during slow walking in vivo, although admittedly our small sample sizes preclude conclusive detection of any statistically significant differences.

Our kinetic analysis showed that the stainless steel shoe may shift craniocaudal, mediolateral and vertical GRFs over much of stance, which is in accord with previous studies in Thoroughbred (Roepstorff, Johnston \& Drevemo 1999) and Warmblood horses (Willeman, Savelberg \& Barneveld 1998). The reported differences in GRFs between the shod and unshod horse may be due to the grip or impact attenuation properties of the shoe material. Previous studies have reported that horseshoe materials have variable frictional and damping properties and can affect the dynamics of the foot in horses (Heidt et al., 1996; Wilson et al., 1992; Pardoe et al., 2001). It is thus possible that an increase in the craniocaudal GRF may be due to the gripping properties of the steel shoe when in contact with the experimental platform, which could shorten the slip time and increase musculoskeletal forces after impact (Willemen 1997; Johnston et al., 1995).

Using XROMM we accurately measured in vivo joint kinematics data of the Thoroughbred horse under shod and unshod conditions without artefacts from the skin and other underlying soft tissue motions. The results were consistent with our expectations for a cursorial animal such as our horse subject. During the stance phase in both shod and unshod conditions, the horse's forefoot joints extended by large amounts but minimal motion occurred in adduction-abduction and longitudinal rotation. This finding corresponds to those from previous kinematic studies on unshod horses during walking that also reported flexion-extension as the dominant rotation and only minimal adduction-abduction and longitudinal rotations (Clayton et al., 2007a; Clayton et al., 2007b). The negligible rotational differences between the shod and the unshod conditions during longitudinal rotation and adduction-abduction found in our study likely are confounded 
462 not only by our small sample sizes but also by noise due to the very small rotations and human 463 error in rotoscoping such fine details of motion. Menegaz et al.'s (2015) kinematic study on pig 464 feeding also attributed minimal rotations that failed to pass their precision threshold to noise 465 introduced by the XROMM analysis procedure. An additional issue of uncertainty with our 466 subject is whether it had any foot balance issues, either in terms of clinical observations or centre 467 of pressure patterns, but available information do not allow us to assess this possibility.

468 Our kinematic data for both the shod and unshod conditions showed flexed postures in both 469 the PIP and DIP joints from approximately 10\% of stance until mid-stance with maximal joint 470 extension occurring just before the foot leaves the ground. This finding is consistent with 471 previous studies of horse foot kinematics in both walking and trotting, which have shown that the 472 PIP and DIP joints maintain a similar motion pattern in those gaits, with changes evident only in 473 the amounts of rotation (Chateau, Degueurce \& Denoix 2004; Clayton et al., 2007b). The greater 474 extension of the DIP joint relative to the PIP joint after mid-stance and at late stance is in accord 475 with previous research on walking and trotting horses in shod and unshod conditions (Clayton et 476 al., 2007b; Roach et al., 2015; Roepstorff, Johnston \& Drevemo 1999). Although the horse 477 received training prior to data collection, walking on a platform surrounded by equipment could have intimidated the animal and thereby influenced its natural locomotor behaviour and speed. Future research should measure more individuals to account for intraspecific variations in locomotor behaviours.

A constraint on this sort of multi-individual study, however, is that each individual must have its distal limb CT or MRI scanned to obtain subject-specific morphological data for XROMM 483 analysis, which requires mild sedation, anaesthesia or euthanasia, with accompanying ethical dilemmas and risks, in addition to the very time-intensive nature of not only collecting synchronised kinematic and kinetic data but also processing the XROMM data and subjectspecific musculoskeletal (e.g. OpenSim) and FEA modelling analyses. For this study, we also simplified our implementation of the inverse dynamics technique by assuming a static midstance (i.e. no segment accelerations) posture and that individual foot segments were massless. A sensitivity analysis showed that these assumptions had a negligible $(<1 \%)$ effect on the calculated intersegmental forces and joint torques used in the FEA. However studies of different species and/or motions may invalidate these assumptions and require collecting additional detailed segment inertia and mass properties. Regardless, the inverse dynamics approach 
493 presented here can easily be generalized to account for segment accelerations and gravity, if 494 necessary. Hence, despite our study's restriction to measuring a few steps of one individual and

495

496

497

498

499

500

501

502

503

504

505

506

507

508

509

510

511

512

513

514

515

516

517

518

519

520

521

522

523 assuming a static posture and massless foot segments, it is an important example of the integration of $3 \mathrm{D}$ biomechanical methods and their application to fundamental problems in equine locomotion, care and welfare.

Our FEA results revealed an increase in the von Mises and principal bone stress magnitudes in the shod (vs. unshod) horse's forefoot phalanges at mid-stance. Both conditions showed increased stresses on the distal epiphysis of the proximal phalanx in the dorsal and ventral view (Figure 5-6). The unshod horse showed slightly higher stresses than the shod horse around the sagittal groove of the P1, yet stresses around this area were low compared to the midshaft and the proximal epiphysis. This finding is partly similar to the findings of O'Hare et al., (2013) for walking, yet their study found higher stresses around the sagittal groove of the proximal phalanx. This is potentially due the fact that the proximal-distal force that was assigned to the O'Hare et al., (2013) model to simulate walking was $3600 \mathrm{~N}$, but our inverse dynamics analysis results were $2503 \mathrm{~N}$ and $2354 \mathrm{~N}$ for the shod and unshod conditions respectively. In addition our model is solid, stiffer and thus will have smaller responses to stress.

Our FE analysis also showed an increase in von Mises and principal stresses around the midshaft of the intermediate phalanx and around the proximal borders of the navicular bone. However, in both the shod and unshod conditions, stresses around the navicular bone were minimal. A more advanced model that includes the superficial and deep digital flexor tendons is required to assess whether the navicular bone acts more like a lever for the deep digital flexor tendon (DDFT) (Eliashar, McGuigan and Wilson 2004), rather than in direct load-bearing.

A major issue in subject-specific FE models when based on in vivo experiments is the location of the constraints on the bones. We here showed that expansion of the surface area of the constrained nodes on the palmar aspect of the P3 reduced the magnitudes and patterns of stresses dorsally but it did not affect the overall comparisons between the shod and unshod conditions. In all cases, the shod horse showed higher concentration of stresses on the P3 than the unshod condition, with the caveat that none of these differences were statistically tested.

Boundary conditions are an area of uncertainty in FEA studies of long and short bones when in vivo conditions are simulated. In this study we applied our loads to the entire surface of the joint of interest and we constrained all nodes on the distal joint surface for the P1 and P2. Our 
524 approach may have over-constrained the models, but is reproducible when FEA is conducted on 525 bones of varying shape and size. An alternative approach would be to only constrain the node 526 that is located along the same centroid axis with the load centroid, and constrain the rest of the 527 nodes only on the long axis of the bone. This modelling approach would also allow compression 528 whilst preventing bending but it is not known if, biologically, the joints are loaded and 529 constrained along their long axes so precisely. It is also not feasible to define a node on the exact 530 centroid axes on a mesh file of a bone when the geometry is not symmetrical. Our sensitivity 531 analysis of the boundary conditions applied to the P1 showed that our modelling approach 532 reduces stresses on the distal end of the $\mathrm{P} 1$, but in both cases bending occurred craniocaudally 533 (Figure S7).

$534 \quad$ FEA is a modelling approach and inevitably will introduce modelling errors/artefacts. In a 535 comparative context (e.g. shod vs. unshod) it is unlikely that modelling artefacts will affect the 536 ultimate comparisons, however rigorous sensitivity analyses are recommended for clinical 537 applications when absolute stress magnitudes are essential.

538 There is also the valid concern that, although our experimental data (kinematics and 539 kinetics) are in vivo measurements of real motions and have a high degree of precision, our 540 OpenSim (i.e., inverse dynamics) and FEA modelling analyses did not account for the tissues of 541 the hooves themselves, the shoes, ligaments, tendons, frog or other soft tissues that would 542 certainly alter the mechanics of the foot. Thus our analysis shows what the influence of shod vs. 543 unshod conditions of our horse subject were solely upon the in vivo dynamics (including the 544 altered GRFs and motions) and upon the stresses within the bones in the theoretical case of those 545 bones bearing all loads themselves. Certainly the absolute values of the stresses would change 546 with the addition of soft tissue data and neuromuscular control, but it is less certain how much 547 the relative stresses would change between the shod vs. unshod conditions. Regardless, this will 548 remain unknown until more sophisticated models are created and additional studies are 549 conducted. Even so, we have presented the first analysis that integrates state-of-the-art methods 550 for kinematic and kinetic analysis with musculoskeletal modelling and finite element analysis 551 methods for the distal foot of horses, which itself is a considerable methodological advance that 552 future studies can build upon.

553 Our preliminary study illustrates that the stainless steel shoe may influence the dynamics 554 and mechanics of a Thoroughbred horse's forefoot during slow walking, although our results are 
555 inconclusive in some important aspects. Certainly, more research is needed to quantify the effect

556 of the shoe on the equine forefoot during the whole stance phase, under different trimming

557 protocols, at faster speeds/gaits and with more individuals and strides as well as a similar focus

558 on the hindfeet. Expansion of this research question, especially via the application of this novel

559 combination of in vivo experiments and computer models should not only create a foundation of

560 stronger data and inferences on which future studies can continue to build on, but can also

561 bolster confidence in equine biomechanics to better understand the form, function and

562 pathological relationships of the anatomical tissues of the equine foot.

563

\section{Acknowledgements}

565 We thank Sharon Warner, Emil Olsen, Renate Weller, Luis Lamas, Emily Sparkes, Heather 566 Paxton and Julia Molnar for their assistance and technical support during data collection. We 567 also thank Phil Pickering for his assistance with the setup of the custom-made platform and the 568 fluoroscopy system and Justin Perkins for allowing us access to the Thoroughbred horse. We 569 thank Jan Janzekovic and Hyab Mehari Abraha from the Moving Morphology \& Functional 570 Mechanics Laboratory for their assistance in data analysis. We are grateful to Sandra Shefelbine 571 and Andrea Pereira for useful discussions on FEA and our colleagues at the University of Brown

572 (Sabine Moritz, David Baier and Beth Brainerd) for their endless support during the processing 573 of the XROMM data. Particular thanks are also due to Todd Pataky and Vivian Allen for 574 valuable discussions. Finally, we appreciate the constructive criticisms of our Editor and our 3 575 reviewers.

576

\section{References}

578 Alexander RMcN, Jayes AS. 1983. A dynamic similarity hypothesis for the gaits of quadrupedal 579 mammals. Journal of Zoology 201:135-152. DOI: 10.1111/j.1469-7998.1983.tb04266.x

Astley HC, Roberts TJ. 2012. Evidence for a vertebrate catapult: elastic energy storage in the plantaris tendon during frog jumping. Biology Letters 8(3):386-389. three different shoeing conditions on tendon strain in the thoroughbred forelimb. Equine 
590

591

592

593

594

595

596

597

598

599

600

601

602

603

604

605

606

607

608

609

610

611

612

613

614

615

616

617

618

619

620

621

622

623

624

625

626

627

628

629

630

631

632

633

634

Baier DB, Gatesy SM, Dial KP. 2013. Three-Dimensional, High-Resolution Skeletal Kinematics of the Avian Wing and Shoulder during Ascending Flapping Flight and Uphill Flap-Running. PLoS ONE , 8(5): e63982. DOI:10.1371/journal.pone.0063982.

Bailey CJ, Reid SW, Hodgson DR, Rose RJ. 1999. Impact of injuries and disease on a cohort of two- and three year-old thoroughbreds in training. Veterinary Record 145: 487-93.

Bowker RM, Atkinson PJ, Atkinson TS, Haut RC. 2001. Effect of contact stress in bones of the distal interphalangeal joint on microscopic changes in articular cartilage and ligaments. American Journal of Veterinary Research 62:414-424.

Brainerd EL, Moritz S, \& Ritter DA 2015. XROMM analysis of rib kinematics during lung ventilation in the green iguana, Iguana iguana. Journal of Experimental Biology Doi: $10.1242 /$ jeb.127928.

Brainerd EL, Baier DB, Gatesy SM, Hedrick TL, Metzger KA, Gilbert SL, Crisco JJ. 2010. Xray reconstruction of moving morphology (XROMM): precision, accuracy and applications in comparative biomechanics research. Journal of Experimental Zoology. Part A, Ecological Genetics and Physiology 313A: 262-279. DOI:10.1002/jez.589

Camp AL, Roberts TJ, Brainerd EL. 2015. Swimming muscles power suction feeding in largemouth bass. PNAS 112: 8690-8695.

Camp AL, Brainerd EL. 2014. Role of axial muscles in powering mouth expansion during suction feeding in largemouth bass (Micropterus salmoides). Journal of Experimental Biology 217: $1333-45$.

Chateau H, Degueurce C, Denoix J-M. 2004. Evaluation of three-dimensional kinematics of the distal portion of the forelimb in horses walking in a straight line. American Journal of Veterinary Research 65: 447-455.

Clayton HM, Sha DH, Stick JA, Elvin N. 2007a. 3D kinematics of the equine metacarpophalangeal joint at walk and trot. Veterinary and Comparative Orthopaedics and Traumatology 20: 86-91.

Clayton HM, Sha DH, Stick JA, Robinson P. 2007b. 3D kinematics of the interphalangeal joints in the forelimb of walking and trotting horses. Veterinary and Comparative Orthopaedics and Traumatology 20: 1-7.

Clayton HM, Sha DH, Stick JA, Mullineaux DR. 2004. Three dimensional kinematics of the equine carpus at trot. Equine Veterinary Journal 36: 671-676.

Clegg PD. 2011. Musculoskeletal disease and injury, now and in the future. Part 1. Fractures and fatalities. Equine Veterinary Journal 43 (6): 643-649. 
635 Cogger N, Evans D, Perkins N, Hodgson D, Reid S. 2006. Risk Factors for injuries in 636 Thoroughbred Horses. A report for the Rural Industries Research and Development Corporation. 637 Australia Government, Rural Industries Research and Development Corporation RIRDC 638 Publication No 06/050.

639

640

641

642

643

644

645

646

647

648

649

650

651

652

653

654

655

656

657

658

659

660

661

662

663

664

665

666

667

668

669

670

671

672

673

674

675

676

677

678

679

680

Colles C. 1989. The relationship of frog pressure to heel expansion. Equine Veterinary

Journal 21: 13-16.

Collins SN, Murray RC, Kneissl S, Stanek C, Hinterhofer C. 2009. Thirty-two component finite element models of a horse and donkey digit. Equine Veterinary Journal 41(3): 219-224.

Dawson MM, Metzger KA, Baier DB, Brainerd EL. 2011. Kinematics of the quadrate bone during feeding in Mallard ducks. Journal of Experimental Biology 214: 2036-2046.

Delp SL., Anderson FC, Arnold AS, Loan P, Habib A, John C T, Guendelman E, Thelen DG. 2007. OpenSim: Open-Source Software to Create and Analyze Dynamic Simulations of Movement. IEEE Transactions on Biomedical Engineering 54: 11.

Dobbert T. 2005. Matchmoving: the invisible art of camera tracking. Alameda, CA: Sybex

Douglas JE, Biddick TL, Thomason JJ, Jofriet JC. 1998. Stress/strain behaviour in the laminar junction. Journal of Experimental Biology 201:2287-2297.

Dyce KM, Sack WO, Wensing CJG. 2010. Textbook of Veterinary Anatomy (fourth edition). Saunders Elsevier

Dyhre-Poulsen P, Smedegaard HH, Roed J, Korsgaard E.1994. Equine hoof function investigated by pressure transducers inside the hoof and accelerometers mounted on the first phalanx. Equine Veterinary Journal 26:362-366.

Eliashar E, McGuigan MP, Wilson AM. Relationship of foot conformation and force applied to the navicular bone of sound horses at the trot 2004. Equine Veterinary Journal 36:431-435.

Falkingham PL, Gatesy SM. 2014. The birth of a dinosaur footprint: Subsurface 3D motion reconstruction and discrete element simulation reveal track ontogeny. PNAS 111: 18279-18284.

Foote C, McIntosh A, V'Landys P, Bulloch K. 2011. Health and Safety in Australian Horse Racing. Canberra, Australia: ACT

Gatesy SM, Baier DB, Jenkins FA, Dial KP. 2010. Scientific rotoscoping: a morphology-based method of 3-D motion analysis and visualization. Journal of Experimental Zoology Part A: Ecology Genetics and Physiology 313A: 244-261.

Gidmark NJ, Konow N, LoPresti E, Brainerd EL 2013. Bite force is limited by the force-length relationship of skeletal muscle in black carp, Mylopharyngodon piceus. Biology Letters 9:20121181. 
681

682

683

684

685

686

687

688

689

690

691

692

693

694

695

696

697

698

699

700

701

702

703

704

705

706

707

708

709

710

711

712

713

714

715

716

717

718

719

720

721

722

723

724

725

726

Harrison SM, Whitton RC, Kawcak CE, Stover SM, Pandy MG. 2014. Evaluation of a subjectspecific finite-element model of the equine metacarpophalangeal joint under physiological load. Journal of Biomechanics, 47: 65-73.

Heers AM, Baier DB, Jackson BE, Dial KP. 2016. Flapping before flight: High resolution, threedimensional skeletal kinematics of wings and legs during avian development. PlosONE DOI:10.1371/journal.pone.0153446.

Heidt RSJr, Dormer SG, Cawley PW, Scranton PE Jr, Losse G, Howard M. 1996. Differences in frictional and torsional resistance in athletic shoe-turf interfaces. American Journal of Sports Medicine 24:834-842.

Hinterhofer C, Stanek C, Haider H. 2001. Finite element analysis (FEA) as a model to predict effects of farriery on the equine hoof. Equine Veterinary Journal 33:58-62.

Hinterhofer C, Stanek C, Binder K. 1998. Elastic modulus of equine hoof horn, tested in wall samples, sole samples and frog samples at varying levels of moisture. Berl Munchener Tierarzt Wochenschrift 111:217-221.

Hutton DV. 2003. Fundamentals of finite element analysis. McGraw-Hill Inc. New York.

Jansová M, Ondoková L, Vychytil J, Kochová P, Witter K, Tonar Z. 2015. A finite element model of an equine hoof. Journal of Equine Veterinary Science 35: 60-69.

Jeffcott LB, Rossdale PD, Freestone J, Frank CJ, Towers-Clark PF. 1982. An assessment of wastage in thoroughbred racing from conception to 4 years of age. Equine Veterinary Journal 14: $185-198$.

Johnston C, Roepstorff L, Drevemo S, Kallings P. 1995. Kinematics of the distal forelimb during the stance phase in the fast trotting Standardbred. Equine Veterinary Journal, Suppl. 18: $170-174$.

Jordan P, Willneff J, D‘Apuzzo N, Weishaupt M, Wistner T, Auer J. 2001. Photogrammetric measurement of deformations of horse hoof horn capsules. In Videometrics and optical methods for $3 D$ shape measurements, Proc SPIE, 4309: 204-11.

Kambic RE, Roberts TJ, Gatesy SM. 2015. Guineafowl with a twist: asymmetric limb control in steady bipedal locomotion. Journal of Experimental Biology. 218: 3836-3844.

Kambic RE, Roberts TJ, Gatesy SM. 2014. Long-axis rotation: a missing degree of freedom in avian bipedal locomotion. Journal of Experimental Biology. 217: 2770-2782.

Lundberg A, Goldie I, Kalin B, Selvik G. 1989. Kinematics of the ankle/foot complex: plantarflexion and dorsiflexion. Foot \& Ankle International 9:194-200. 
727 McClinchey HL, Thomason JJ, Jofriet JC. 2003. Isolating the effects of equine hoof shape 728 measurements on capsule strain with finite element analysis. Veterinary and Comparative 729 Orthopaedics and Traumatology 16:67-75.

730

McKee SL. 1995. An update on racing fatalities in the UK. Equine Veterinary Education 7(4): 732 202-204.

733

734

Menegaz RA, Baier DB, Metzer KA, Herring SW, Brainerd EL. (2015). XROMM analysis of

735 tooth occlusion and temporomandibular joint kinematics during feeding in juvenile miniature pigs. Journal of Experimental Biology 218:2573-2584. DOI: 10.1242/jeb.119438

Miranda DL, Rainbow MJ, Crisco JJ, Fleming BC. 2013. Kinematic differences between optical motion capture and biplanar videoradiography during a jump-cut maneuver. Journal of Biomechanics 46(3):567-573.

Moyer W, Anderson JP. 1975. Lameness caused by improper shoeing. Journal of American Veterinary Medical Association 166:47-52.

Nowroozi B, Brainerd EL. 2013. X-ray motion analysis of the vertebral column during the startle response in striped bass, Morone saxatilis. Journal of Experimental Biology, 216: 2833-2842

Nyakature JA, Fischer MS. 2010. Three-dimensional kinematic analysis of the pectoral girdle during upside-down locomotion of two-toed sloths (Choloepus didactylus, Linné 1758). Frontiers in Zoology 7:21.

O'Hare LMS, Cox PG, Jeffery N, Singer ER. 2013. Finite element analysis of stress in the equine proximal phalanx. Equine Veterinary Journal DOI: 10.1111/j.2042-306.2012.00635.x.

Pardoe CH, McGuigan MP, Rogers KM, Rowe LL, Wilson AM. 2001. The effect of shoe material on the kinetics and kinematics of foot slip at impact on concrete. Equine Veterinary Journal 33: 70-73.

Parkin TD, Clegg PD, French NP, Proudman CJ, Riggs CM, Singer ER, Webbon PM, Morgan KL. 2004. Risk of fatal distal limb fractures among Thoroughbreds involved in the five types of racing in the United Kingdom. Veterinary Record 154: 493-497.

Reinschmidt C, van den Bogert AJ, Murphy N, Lundberg A, Nigg BM. 1997. Tibiocalcaneal motion during running, measured with external and bone markers. Clinical Biomechanics 12: 816.

Rho JY, Currey JD, Zioupos P, Pharr GM. 2001. The anisotropic Young's modulus of equine secondary osteons and interstitial bone determined by nanoindentation. The Journal of Experimental Biology 204:1774-1781. 
771 Roach JM, Pfau T, Bryars J, Unt V, Channon SB, Weller R. 2015. Sagittal distal limb kinematics 772 inside the hoof capsule captured using high-speed fluoroscopy in walking and trotting horses.

773

774

775

776

777

778

779

780

781

782

783

784

785

786

787

788

789

790

791

792

793

794

795

796

797

798

799

800

801

802

803

804

805

806

807

808

809

810

811

812

813

814

815

816
The Veterinary Journal. DOI: 10.1016/j.tvj1.2014.06.014.

Roepstorff L, Johnston C, Drevemo S. 1999. The effect of shoeing on kinetics and kinematics during the stance phase. Equine Veterinary Journal 31 (Suppl. 30): 279-285.

Roepstorff L, Johnston C, Drevemo S. 2001. In vivo and in vitro heel expansion in relation to shoeing and frog pressure. Equine Veterinary Journal 33: 54-57.

Salo Z, Runciman RJ, Thomason JJ. 2009. A dynamic model of a horse hoof at first contact. Biosystems Engineering 103: 364-373.

Thomason JJ, Douglas JE, Sears W. 2001. Morphology of the laminar junction in relation to the shape of the hoof capsule and third phalanx in adult horses (Equus caballus). Cells Tissues Organs 166:304-318.

Thomason JJ, McClinchey HL, Jofriet JC. 2002. The analysis of strain and stress in the equine hoof capsule using finite-element methods: comparison with principal strains recorded in vivo. Equine Veterinary Journal 34:719-725.

Thomason JJ, McClinchey HL, Faramarzi B, Jofriet JC. 2005. Mechanical behavior and quantitative morphology of the equine laminar junction. The anatomical record. Part $A$, Discoveries in molecular, cellular, and evolutionary biology 283(2): 366-79.

van Heel MC, Moleman M, Barneveld A, van Weeren, Back W.2005. Changes in location of centre of pressure and hoof-unrollment pattern in relation to an 8-week shoeing interval in the horse. Equine Veterinary Journal 37: 536-40.

van Heel MC, van Weeren PR, Back W. 2006. Compensation for changes in hoof conformation between shoeing sessions through the adaptation of angular kinematics of the distal segments of the limbs of horses. American Journal of Veterinary Research 67:1199-1203.

van Weeren PR, van den Bogert AJ, Barneveld A. 1990. A quantitative analysis of skin displacement in the trotting horse. Equine Veterinary Journal S9: 101-109.

Wang J, Blackburn TJ. 2000. The AARM/RSNA physics tutorial for residents: x-ray image intensifiers for fluoroscopy. Radiographics 20:1471-1477.

Willemen MA. 1997. Horseshoeing, a Biomechanical Analysis. PhD Thesis, University of Utrecht.

Willemen MA, Savelberg HHCM, Barneveld A. 1998. The improvement of the gait quality of sound trotting warmblood horses by normal shoeing and its effect on the load on the lower forelimb. Livestock Production Science 52: 145-153. 
817 Wilson PD, Ratzlaff MH, Grant BD, Hyde ML, Balch OK. 1992. The effects of a compressible 818 plastic shoe-the Seattle shoe-on the kinematics of the strides of galloping thoroughbred horses. 819 Journal of Equine Veterinary Science 12 (6): 374-381.

820

821

822

823

824

825

826

827

828

829

830

831

832

833

834

835

836

837

838

839

840

841

842

843

844

845

846

847

848

849

Witte TH, Knill K, Wilson AM. 2004. Determination of peak vertical ground reaction force from duty factor in the horse (Equus caballus). Journal of Experimental Biology 207: 3639-3648.

\section{Figure Captions}

Figure 1. A. Experimental set-up of the horse walking on a custom-made platform retrofitted with a force plate and surrounded by the bi-planar fluoroscopy system. B. Virtual setup of the horse right forefoot based on the experimental alignment of the x-ray sources and the intensifiers. Images in black frames (right and left) illustrate the projections of the distal foot from the two x-ray cameras.

Figure 2. XROMM model with bone segments and coordinate systems for the metacarpophalangeal (MCP), proximal interphalangeal (PIP) and distal interphalangeal (DIP) joints. Green, yellow and red arrows represent the $\mathrm{Z}, \mathrm{Y}^{\prime}$ and $\mathrm{X}^{\prime \prime}$ segment axes respectively.

Figure 3. Ground reaction forces normalised to $100 \%$ stance phase for the shod (black lines) and unshod horse (red lines). For the craniocaudal GRF, cranial and caudal are positive and negative respectively. For the mediolateral GRF, medial is positive and lateral is negative. Solid lines represent the trials used in the subsequent finite element analysis.

Figure 4. Degrees of rotation for the proximal interphalangeal (PIP) and distal interphalangeal (DIP) joints, around the flexion (positive) - extension (negative) axes during the stance phase for the shod (black line) and the unshod (red line) conditions. Dotted lines show the individual trials and the bold lines show the mean degrees of rotation for each condition.

Figure 5. Von Mises stress (MPa) distribution results for the shod and the unshod horse foot, in dorsal view. Bones shown from top to bottom are the P1, P2 and P3. Warm (red) and cold (blue) colours show higher and lower von Mises stresses respectively.

Figure 6. Von Mises stress (MPa) distribution results for the shod and the unshod horse foot, in palmar view. Bones shown from top to bottom are the P1, P2 and P3. Warm (red) and cold (blue) colours show higher and lower von Mises stresses respectively.

\section{Supplementary Figures}


850 Figure S1. The position of the custom-designed calibration cube used during the fluoroscopy

851 experiments to calibrate the 3D space in the XROMM analysis.

852 Figure S2. Loading (in red) and boundary (in orange) locations for the P1, P2 and P3 bones (see

853 Methods: Loads and constraints).

854 Figure S3. Maximum principal stress (MPa) distribution results for the shod and the unshod 855 horse foot, in dorsal view. Bones shown from top to bottom are the P1, P2 and P3. Warm (red)

856 and cold (blue) colours show higher and lower maximum principal stresses respectively.

857 Figure S4. Maximum principal stress (MPa) distribution results for the shod and the unshod 858 horse foot, in palmar view. Bones shown from top to bottom are the P1, P2 and P3. Warm (red) 859 and cold (blue) colours show higher and lower maximum principal stresses respectively.

860 Figure S5. Minimum principal stress (MPa) distribution results for the shod and the unshod 861 horse foot, in dorsal view. Bones shown from top to bottom are the P1, P2 and P3. Warm (red) 862 and cold (blue) colours show higher and lower minimum principal stresses respectively.

863 Figure S6. Minimum principal stress (MPa) distribution results for the shod and the unshod 864 horse foot, in palmar view. Bones shown from top to bottom are the P1, P2 and P3. Warm (red) 865 and cold (blue) colours show higher and lower minimum principal stresses respectively.

866 Figure S7.Sensitivity analysis on the P1 constraints. BC1 constraints are applied on the entire 867 distal joint surface of the P1 and fixed in all axes. BC2 constraints involve fix constraining 3 868 nodes at the distal joint surface on the centroid axis of the load. The remainder nodes are fixed 869 on the long axis of the bone only. Transparent and non-transparent images represent the 870 undeformed and deformed bone respectively. Warm (red) and cold (blue) colours show higher 871 and lower von Mises stresses respectively. Grey colours show von Mises stresses with 872 magnitudes larger than $60 \mathrm{MPa}$.

873 Figure S8. Sensitivity analysis on the location of the P3 constraints and the respective von Mises 874 stresses patterns on the palmar and dorsal views. Warm (red) and cold (blue) colours show

875 higher and lower von Mises stresses respectively.

877 Supplementary Movies

878 Movie S1. Animation of the shod horse during walking.

879 Movie S2. Animation of the unshod horse during walking. 880 


\section{Supplementary Data Captions}

882 Supplementary Data 1. Raw speed data for the unshod $(n=4)$ and shod $(n=4)$ conditions. 883 Column A shows the conditions. Column B lists the name and date of the steps. Column C lists 884 the horse's hip height in meters. Column D lists the frame rate of the Sony camera used for the 885 speed calculations. Columns E and F list the start and end frame of each trial and each condition. 886 Column G shows the difference between the start and end frame (i.e. number of frames elapsed). 887 Column $\mathrm{H}$ shows the time in seconds and was calculated by dividing 1 over the camera frame 888 rate (column D), multiplied by the frame difference (column G). Column I shows the distance 889 that a marker placed on the middle of the body of the horse travelled between the start and end 890 frames of the steps (columns E and F). Column J lists the velocity calculations per trial and 891 condition. Velocity was measured by dividing the distance (column I) over the time (column H). 892 Column K lists gravity at $9.81 \mathrm{~ms}^{-2}$ and column L lists the Froude number per trial and condition. 893 Rows J6 and J 12 show the average velocity for the unshod and shod condition respectively. 894 Rows L6 and L12 show the average Froude number for the unshod and shod conditions 895 respectively.

896

897 Supplementary Data 2. Ground reaction force (GRF) data in Newtons for the unshod $(n=4)$ and 898 shod $(\mathrm{n}=4)$ conditions.

899

900 Supplementary Data 3. Degrees of motion for proximal interphalangeal (PIP) and distal 901 interphalangeal (DIP) joints for the shod $(n=4)$ and unshod $(n=4)$ conditions about the flexion902 extension axis. 


\section{Table $\mathbf{1}$ (on next page)}

Minimum (Min), maximum (Max) and mean Froude number and velocity data, with standard deviation (SD), for the shod $(n=4)$ and unshod $(n=4)$ horse trials. 
1 Table 1. Minimum (Min), maximum (Max) and mean Froude number and velocity data, with

2 standard deviation (SD), for the shod $(n=4)$ and unshod $(n=4)$ horse trials.

\begin{tabular}{lrrrrr}
\hline Condition & $n$ & \multicolumn{3}{c}{ Froude number } \\
\cline { 3 - 6 } & & Min & Max & Mean & SD \\
\hline SHOD & 4 & 0.045 & 0.076 & 0.058 & 0.013 \\
UNSHOD & 4 & 0.060 & 0.069 & 0.064 & 0.0045 \\
\hline Condition & $n$ & \multicolumn{3}{c}{ Velocity $\left(\mathbf{m s}^{-1}\right)$} \\
\cline { 3 - 6 } & & Min & Max & Mean & SD \\
\hline SHOD & 4 & 0.64 & 0.83 & 0.72 & 0.079 \\
UNSHOD & 4 & 0.74 & 0.79 & 0.76 & 0.024 \\
\hline
\end{tabular}

3

4 


\section{Table 2 (on next page)}

Mean degrees of rotation for the proximal interphalangeal (PIP) and distal interphalangeal (DIP) joints of the shod and unshod conditions during the stance phase.

Note that none of these differences can be considered statistically significant. 
1 Table 2. Mean degrees of rotation for the proximal interphalangeal (PIP) and distal

2 interphalangeal (DIP) joints of the shod and unshod conditions during the stance phase. Note that 3 none of these differences can be considered statistically significant.

\begin{tabular}{r|rrrr} 
& \multicolumn{2}{c}{ PIP } & \multicolumn{2}{c}{ DIP } \\
\hline \% Stance & UNSHOD & SHOD & UNSHOD & SHOD \\
\hline 10 & 5.1 & 6.3 & 6.4 & 3.5 \\
15 & 5.2 & 5.8 & 6.7 & 4.1 \\
20 & 4.6 & 4.7 & 6.3 & 4.4 \\
25 & 3.5 & 3.3 & 5.1 & 4.4 \\
30 & 2.4 & 1.8 & 3.5 & 3.8 \\
35 & 1.3 & 0.7 & 1.6 & 3.1 \\
40 & 0.3 & -0.5 & -0.3 & 1.9 \\
45 & -0.6 & -1.8 & -2 & 0.1 \\
50 & -1.5 & -2.9 & -3.6 & -2.8 \\
55 & -2.4 & -3.9 & -5.2 & -6 \\
60 & -3.3 & -5.1 & -6.6 & -9.1 \\
65 & -4.2 & -6.1 & -8.4 & -12.5 \\
70 & -5.4 & -7 & -10.6 & -15.9 \\
75 & -6.4 & -7.5 & -13.7 & -19.3 \\
80 & -7.1 & -7.5 & -18.8 & -22.4 \\
85 & -7.2 & -7.3 & -23.7 & -24.4 \\
90 & -5.8 & -6.8 & -25.7 & -25
\end{tabular}




\section{Table 3(on next page)}

Intersegmental forces in Newtons ( $\mathrm{N}$ ) assigned to the shod and unshod horse finite element models.

Positive values represent forces applied in the distal (i.e. compressive), lateral and cranial directions. 
1 Table 3. Intersegmental forces in Newtons (N) assigned to the shod and unshod horse finite

2 element models. Positive values represent forces applied in the distal (i.e. compressive), lateral

3 and cranial directions.

\begin{tabular}{rlllllr}
\hline & \multicolumn{2}{c}{ Proximal-Distal } & \multicolumn{2}{c}{ Medial-lateral } & \multicolumn{2}{c}{ Cranial-caudal } \\
\hline Force & Shod & Unshod & Shod & Unshod & Shod & Unshod \\
\hline P1 & 2750 & 2413 & -1673 & -1394 & 45 & 198 \\
P2 & 2503 & 2354 & -2024 & -1492 & 48 & 199 \\
& & & & & & \\
P3 & 2580 & 2293 & -1924 & -1583 & 47 & 200
\end{tabular}

4

5

6

7

8 


\section{Figure 1 (on next page)}

Image of experimental and virtual set-up.

A. Experimental set-up of the horse walking on a custom-made platform retrofitted with a force plate and surrounded by the bi-planar fluoroscopy system. B. Virtual setup of the horse right forefoot based on the experimental alignment of the x-ray sources and the intensifiers. Images in black frames (right and left) illustrate the projections of the distal foot from the two x-ray cameras. 

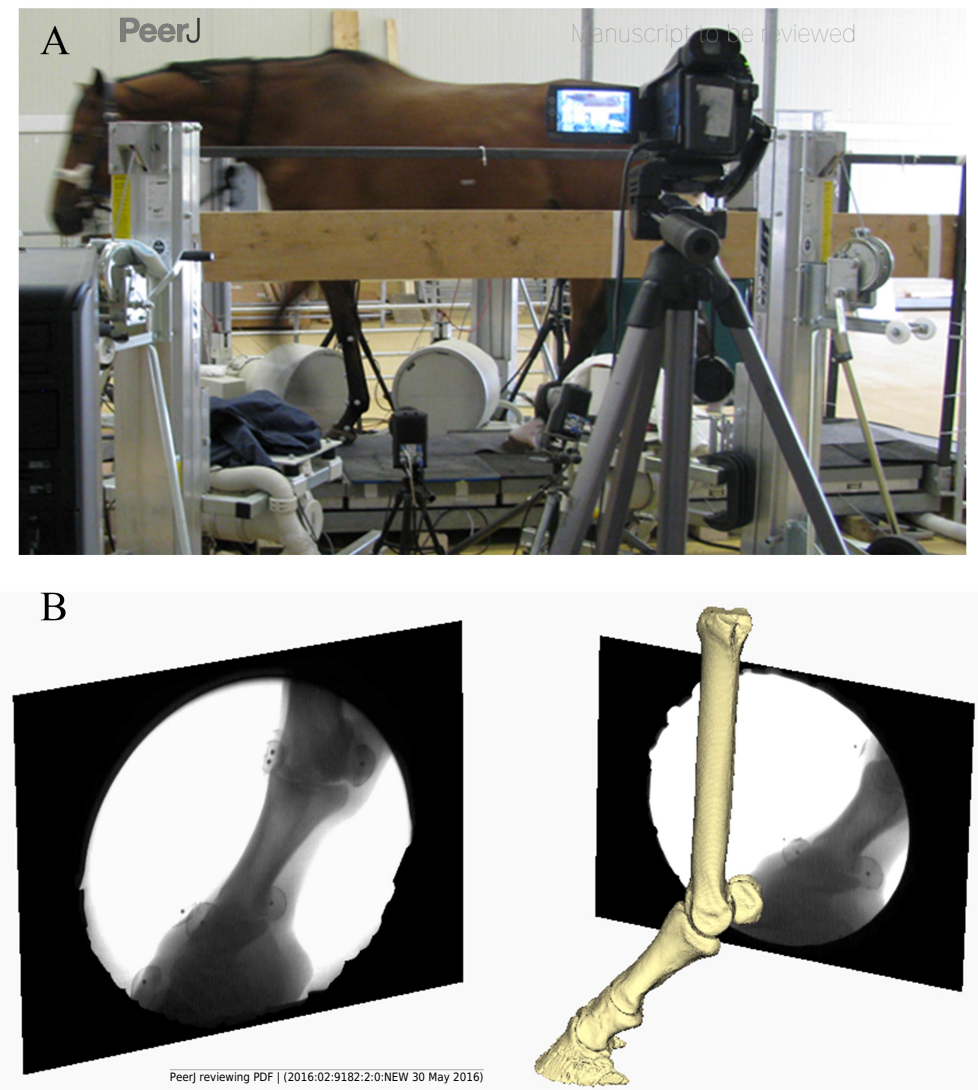
2

XROMM model with bone segments and coordinate systems for the metacarpophalangeal (MCP), proximal interphalangeal (PIP) and distal interphalangeal (DIP) joints.

Green, yellow and red arrows represent the $Z, Y^{\prime}$ and $X^{\prime \prime}$ segment axes respectively. 


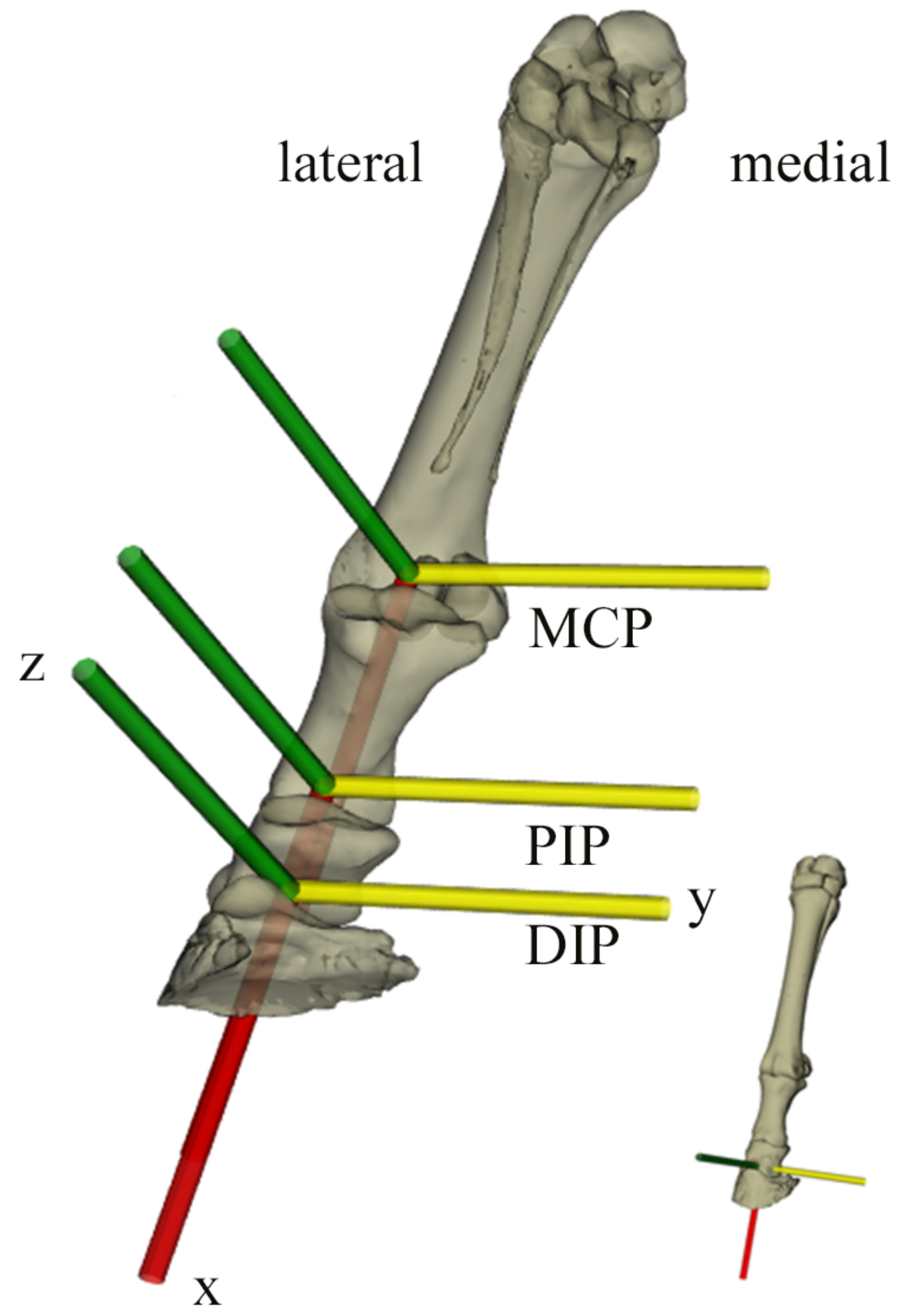


3

Ground reaction forces normalised to $100 \%$ stance phase for the shod (black lines) and unshod horse (red lines).

For the craniocaudal GRF, cranial and caudal are positive and negative respectively. For the mediolateral GRF, medial is positive and lateral is negative. Solid lines represent the trials used in the subsequent finite element analysis. 

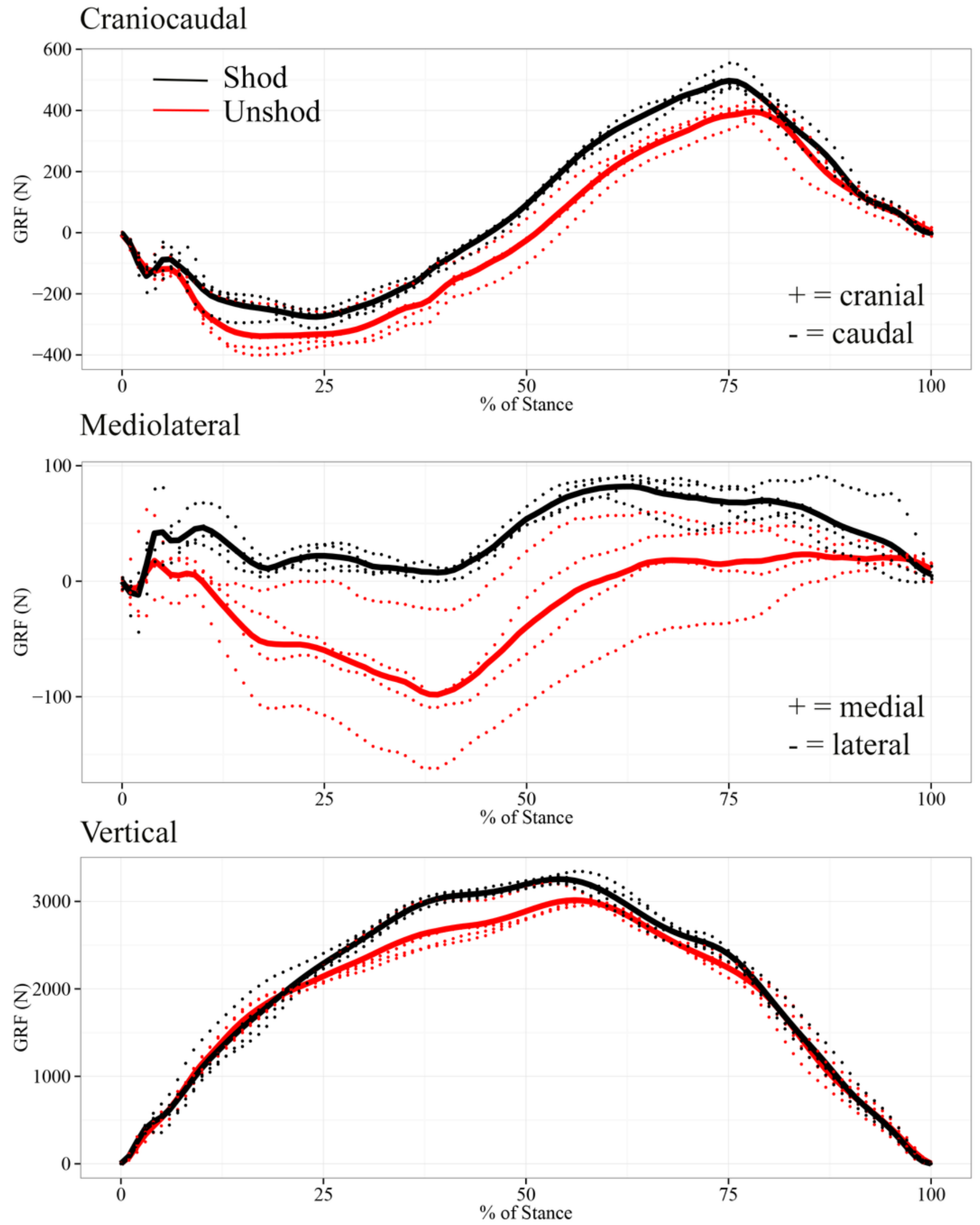


\section{4}

Kinematic data.

Degrees of rotation for the proximal interphalangeal (PIP) and distal interphalangeal (DIP) joints, around the flexion (positive) - extension (negative) axes during the stance phase for the shod (black line) and the unshod (red line) conditions. Dotted lines show the individual trials and the bold lines show the mean degrees of rotation for each condition. 


\section{Flexion-Extension}

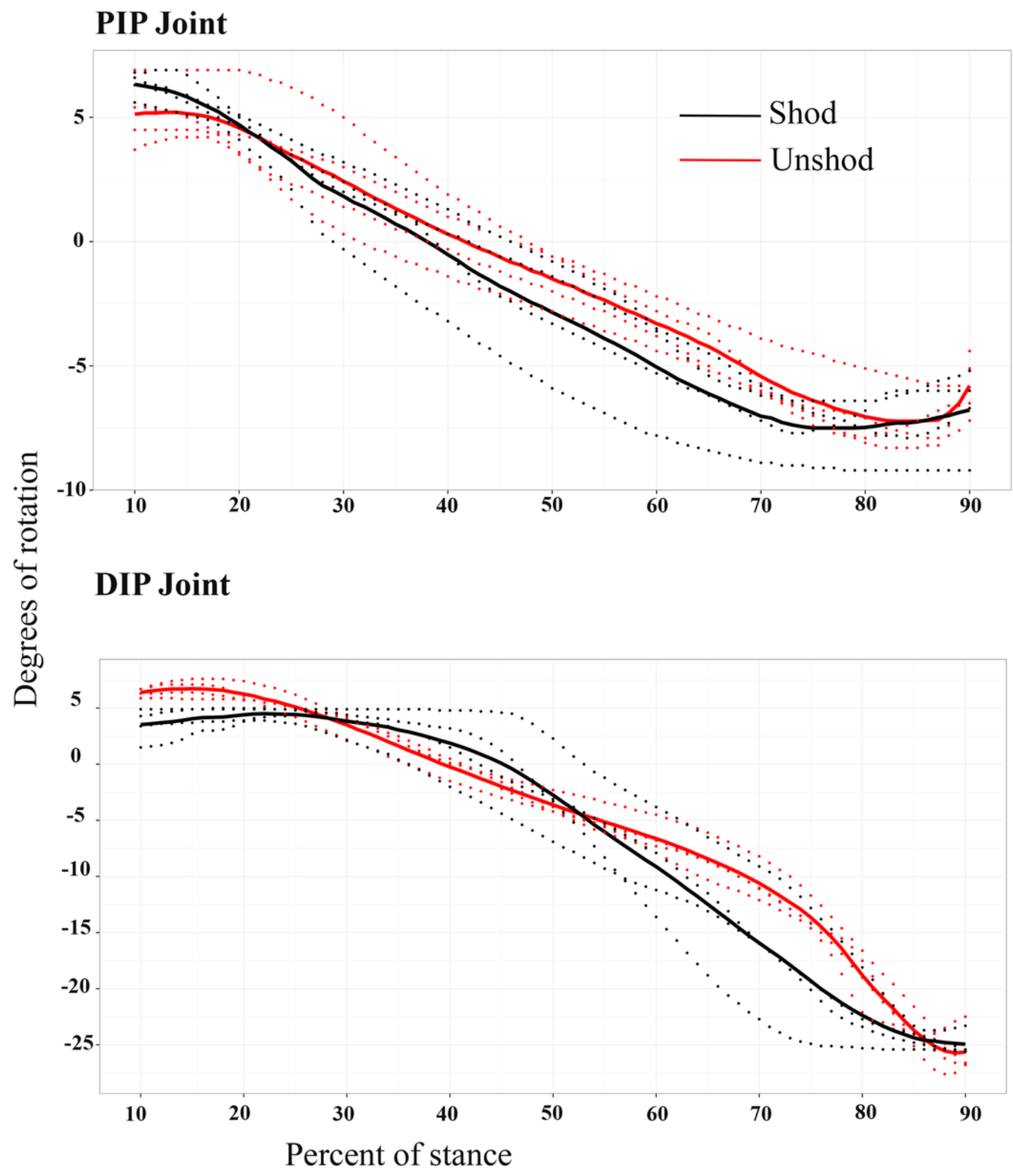




\section{5}

Von Mises stress (MPa) distribution results for the shod and the unshod horse foot, in dorsal view.

Bones shown from top to bottom are the P1, P2 and P3. Warm (red) and cold (blue) colours show higher and lower von Mises stresses respectively.
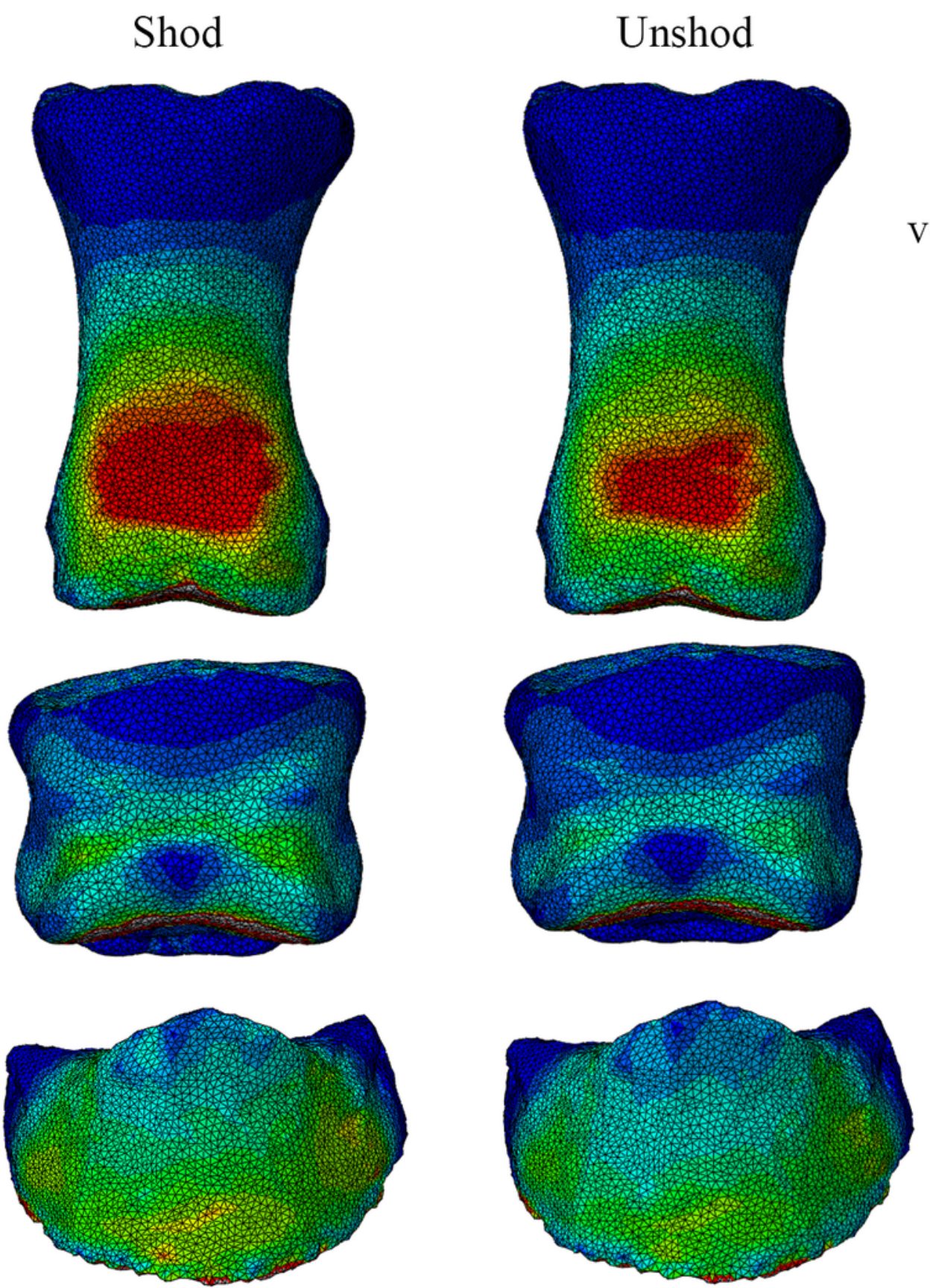

von Mises stress (MPa)

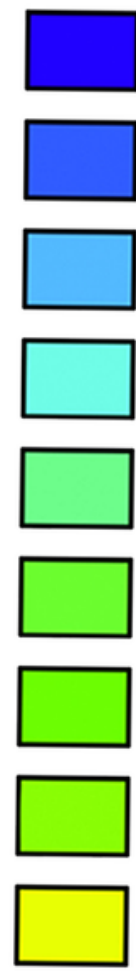

2

4

6

10

12

14

16

20

22

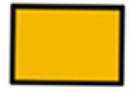

24

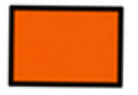

26

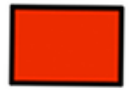

60 
6

Von Mises stress (MPa) distribution results for the shod and the unshod horse foot, in palmar view.

Bones shown from top to bottom are the P1, P2 and P3. Warm (red) and cold (blue) colours show higher and lower von Mises stresses respectively. <?xml:namespace prefix = "o" ns = "urn:schemas-microsoftcom:office:office" />
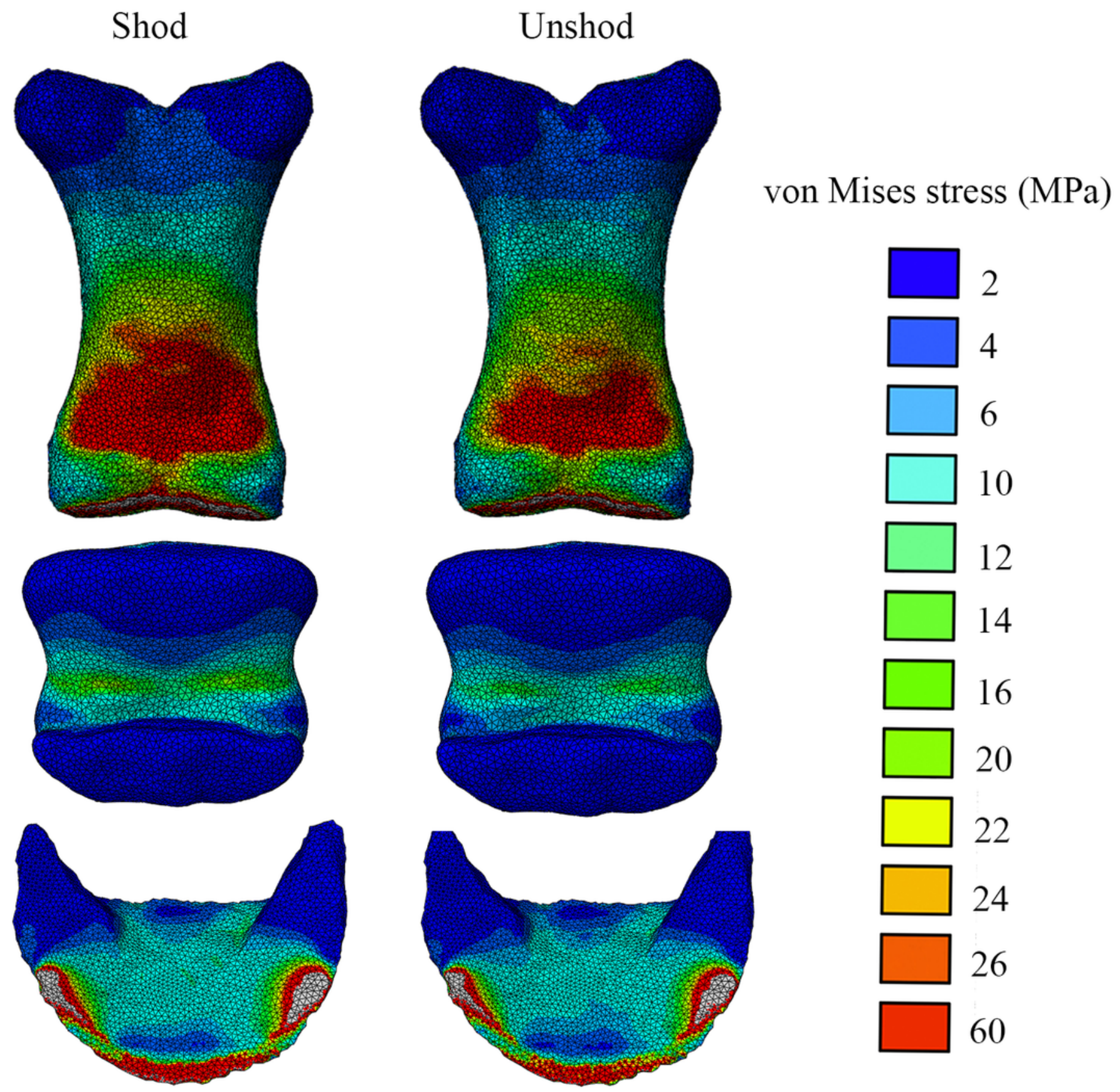

2

4

6

10

12

14

16

20

22

24

26

60 